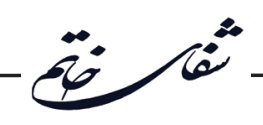

\title{
Stress Detection and Control According to the Skin Signal of Electrical Resistance and Heart Rate Using Reinforcement Learning
}

\author{
Mandana Sadat Ghafourian ${ }^{1 *}$, Amin Noori², Minoochehr Bashizade Fakhar ${ }^{2}$ \\ ${ }^{1}$ Department of Biomedical Engineering, Khaje Nasir Toosi University of Technology, Tehran, Iran \\ ${ }^{2}$ Faculty of Electrical Engineering and Biomedical Engineering, Sadjad University of Technology, Mashhad, Iran
}

$\begin{array}{lll} & \text { Article Info: } & \\ \text { Received: } 24 \text { Jan } 2017 & \text { Revised: } 4 \text { Mar } 2017 & \text { Accepted: } 6 \text { May } 2019\end{array}$

\section{A BSTRACT}

Introduction: The anxiety disorders are the most common mental health condition. Through modulation of stress responses, the anxiety can be controlled. Stress clearly affects the autonomic nervous system and leads to excessive sweating and tachycardia. New methods to identify and manage stress are still needed. Materials and Methods: In this paper, anxiety is controlled by reinforcement learning (RL). For stress level measurement, galvanic skin resistance (GSR) and heart rate were measured. Skin electrical resistance and heart rates are measured in 15 subjects ( 8 females and 7 males), ages between 22 to 30 years, during exposure to melodies and pictures. By connecting bioelectric sensors to the hand, GSR signal can be tested. Anxiety level was measured by biological signals (biofeedback) through playing melodies and showing pictures. First, GSR and heart rate signals were measured (states of $\mathrm{RL}$ ). Then, the application plays a melody (agent) and shows a picture (actions). After a period of time, GSR and heart rate were measured again. Based on the differences between initial and secondary values, the reward, indicating stress variations were calculated. Based on the received reward, the agent learns to control the stress level. Results: The intelligent agent of RL, learns which picture to show and what melody to play in order to manage the stress. For each person, different melodies and pictures reduce the stress. This learning procedure should be repeated for each subject. Conclusion: A novel method is proposed and investigated based on RL for anxiety control. The accuracy of diagnosis is improved in a simple, rapid, and low-cost way.

\section{Key words: \\ 1. Anxiety \\ 2. Skin \\ 3. Heart Rate}

*Corresponding Author: Mandana Sadat Ghafourian

E-mail:m.ghafourian@email.kntu.ac.ir 


\section{تشخيص و كنترل استرس برمبناى سيخنالهاى مقاومت الكتريكى يوست و ضربان قلب با استفاده از يادَيرى تقويتى معاومي}

ماندانا سادات غفوريان '"، امين نورى'، مينوجهر باشى زاده فخار

'كروه مهندسى بزشكى، دانشعاه صنعتى خواجه نصيرالدين طوسى، تهران، ايران

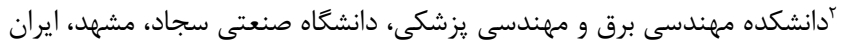

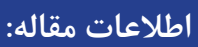

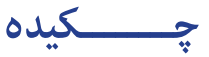

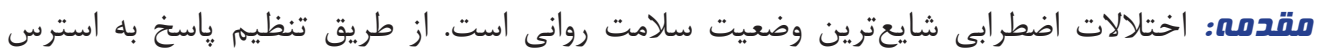

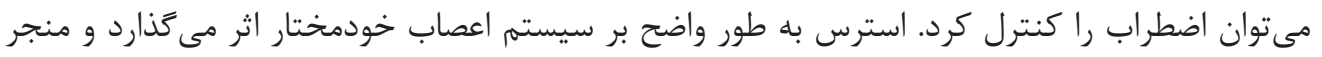

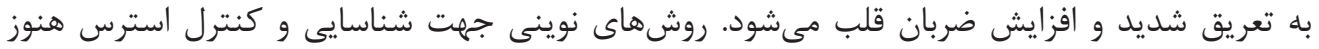

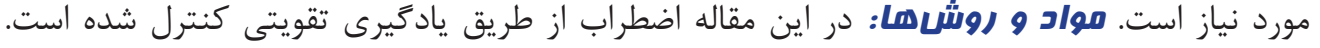

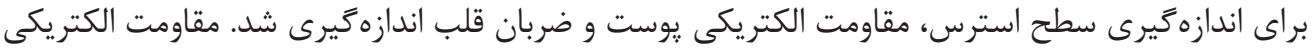

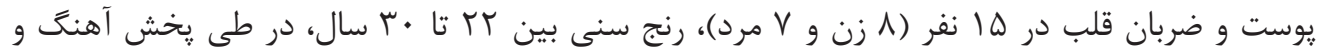

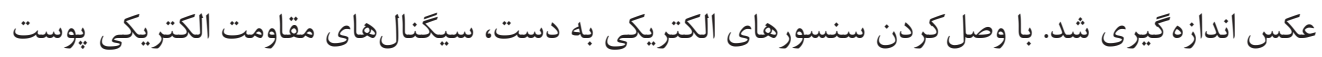

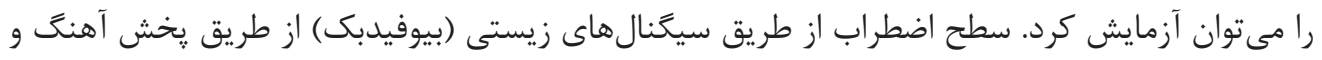

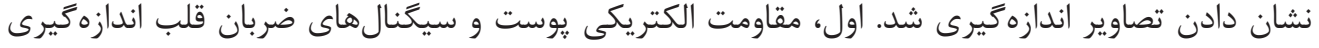

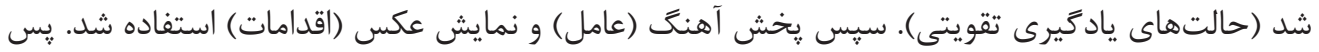

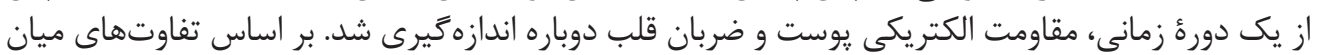

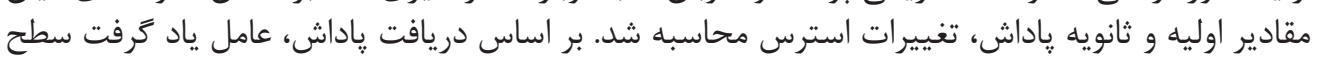

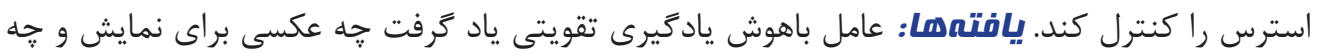

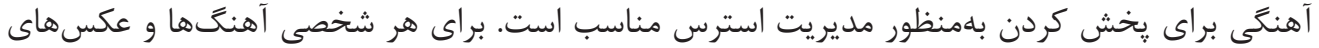

كليد وازهها:

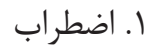

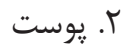

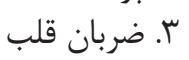

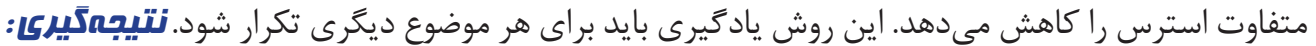

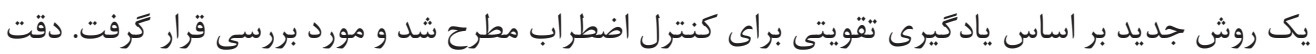

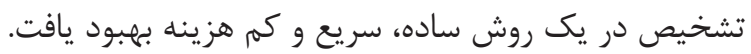




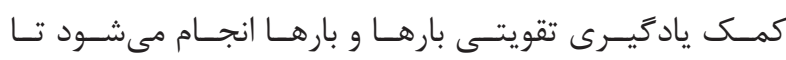

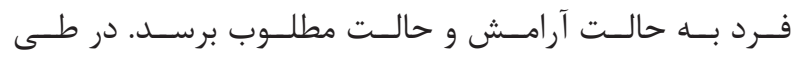

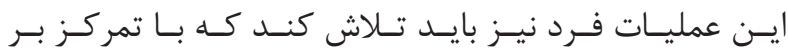

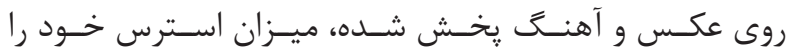

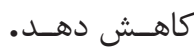

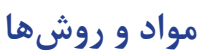

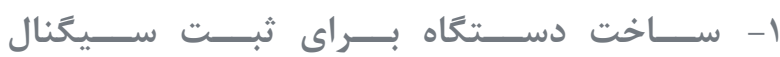

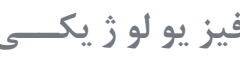

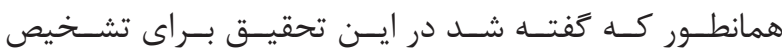

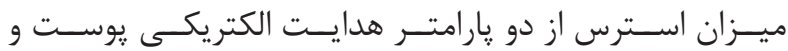

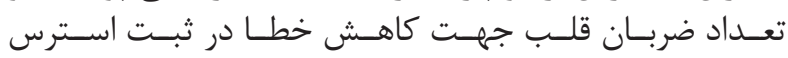

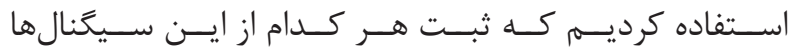

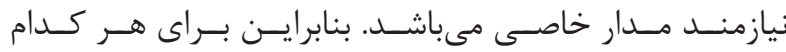

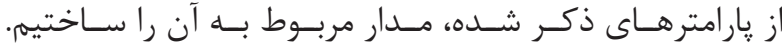

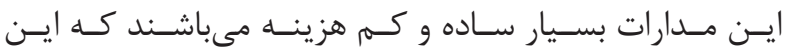

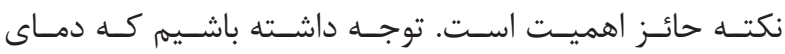

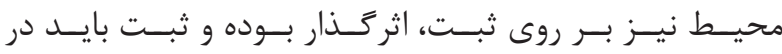

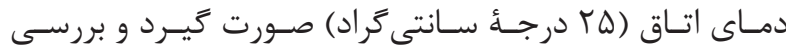

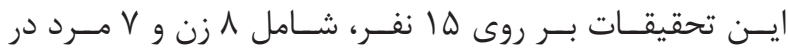

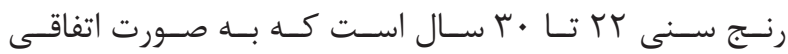

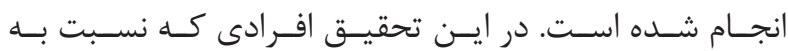

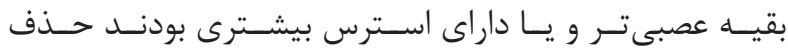

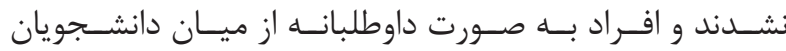

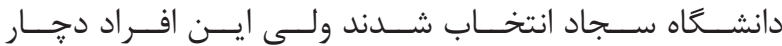

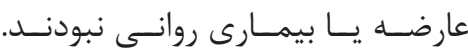

1-1 إمدار تشخيص استرس

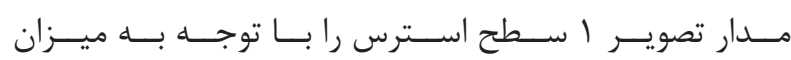

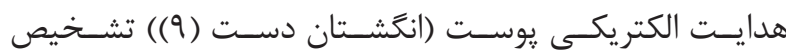

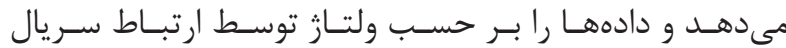

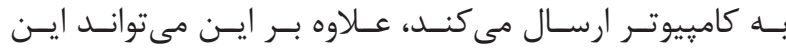

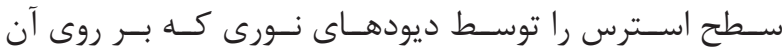

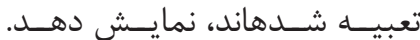

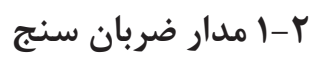

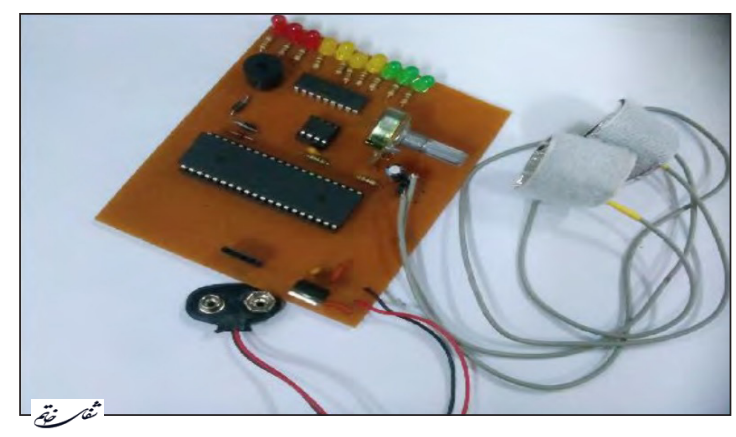

تصوير ا- تصوير گرفته شده از مدار تشخيص استرس در طى ثيروهش.

${ }^{1}$ Pupilometry

${ }^{2}$ Saccadic eye movement

${ }^{3}$ Thermal imaging

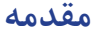

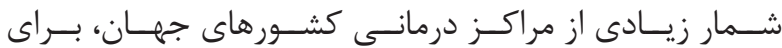

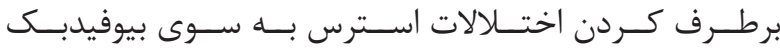

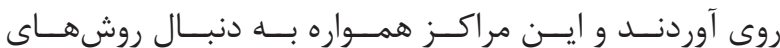

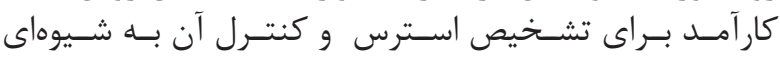

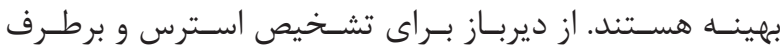

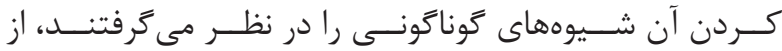

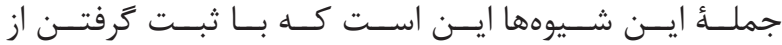

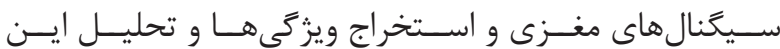

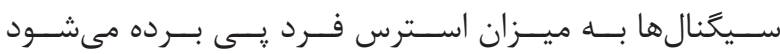

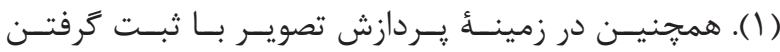

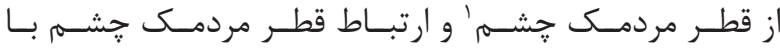

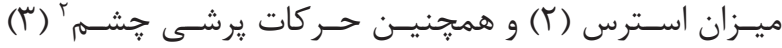

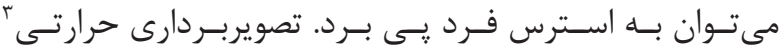

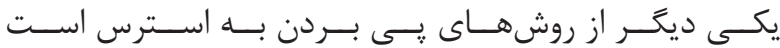

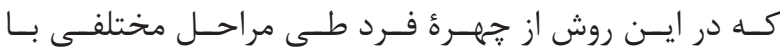

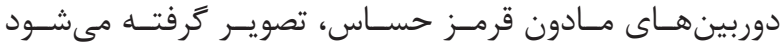

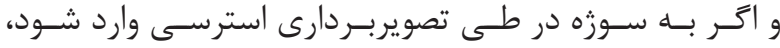

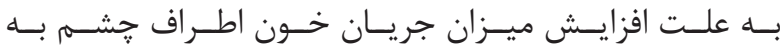

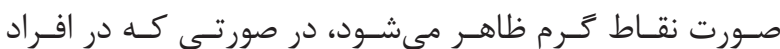

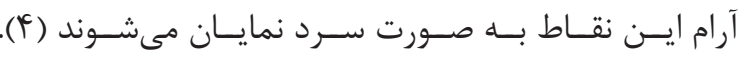

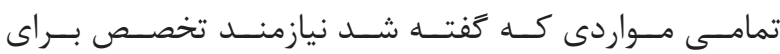

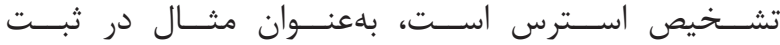

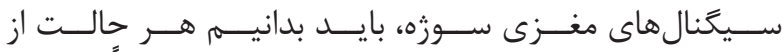

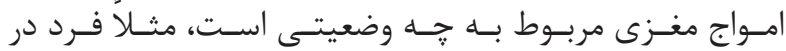

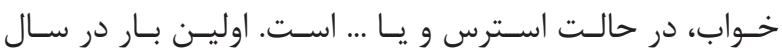

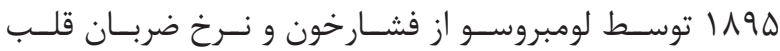

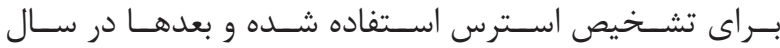

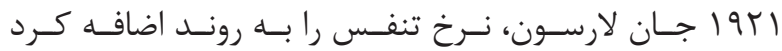

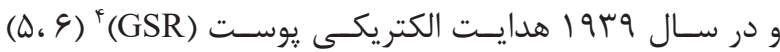

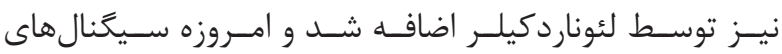

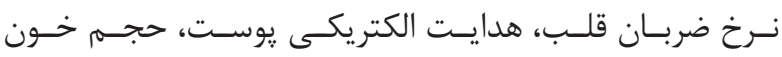

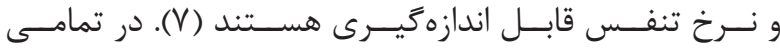

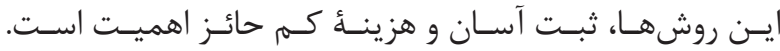

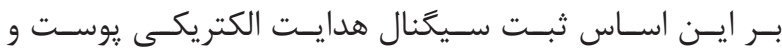

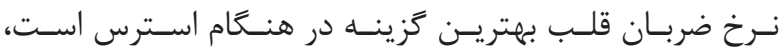

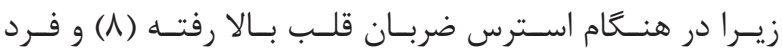

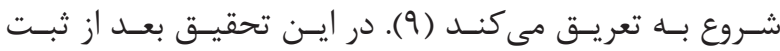

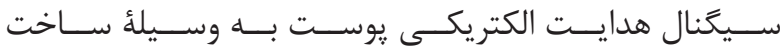

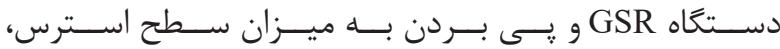

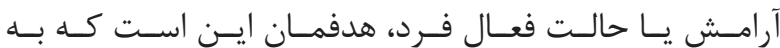

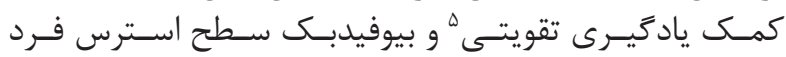

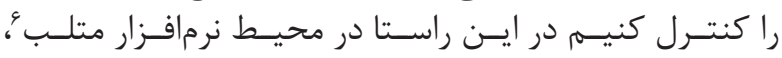

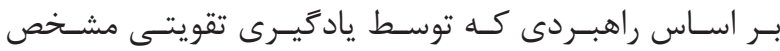

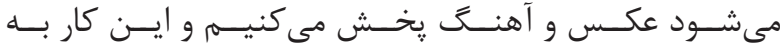

${ }^{4}$ Galvanic skin resistant

${ }^{5}$ Reinforcement learning

${ }^{6}$ Matlab software 
$\mathrm{S}=\{\mathrm{s} 1, \mathrm{~s} 2, \ldots, \mathrm{sn}\}$

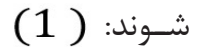

$A=\{a 1, a 2, \ldots, a m\}$

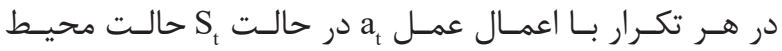

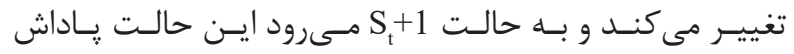

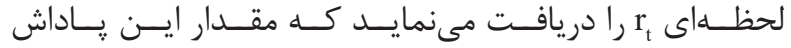

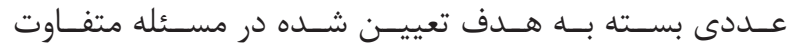

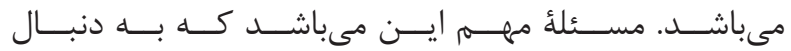

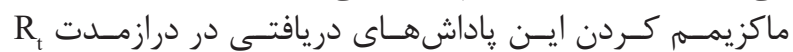

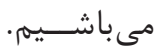

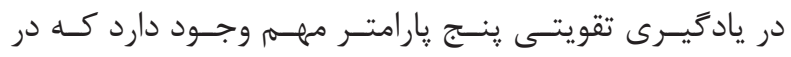

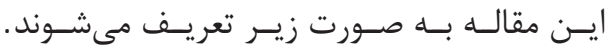

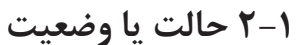

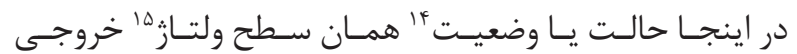

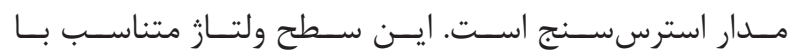

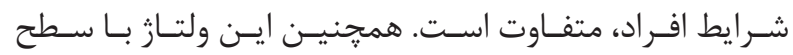

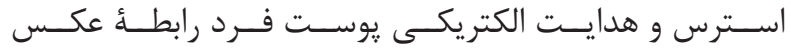

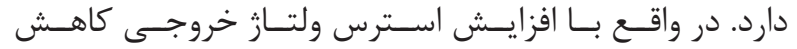

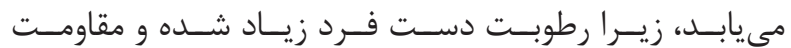

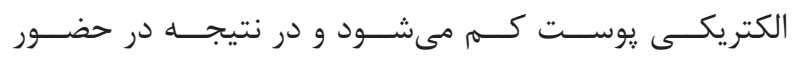

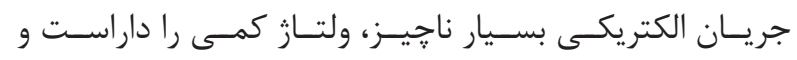

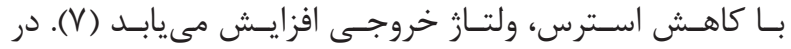

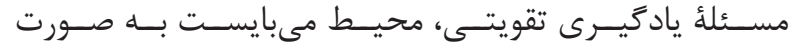

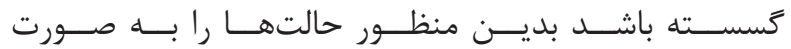

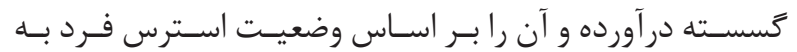

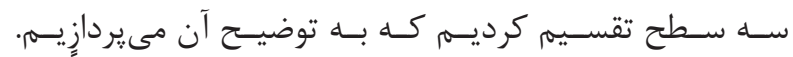

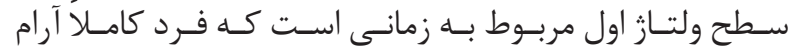

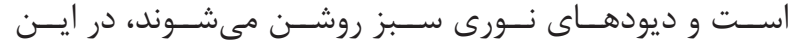

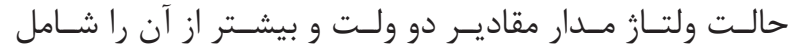

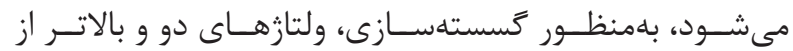

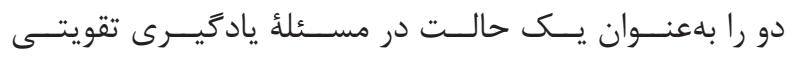

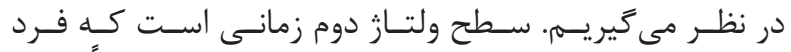

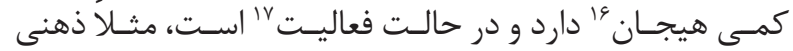

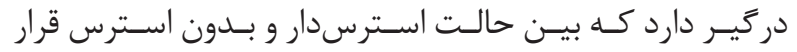

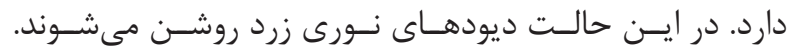

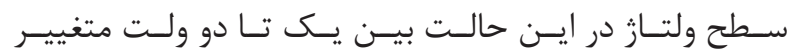

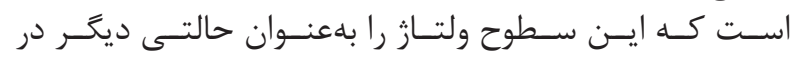

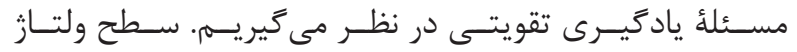

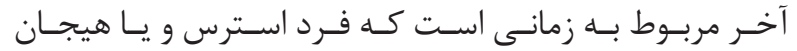

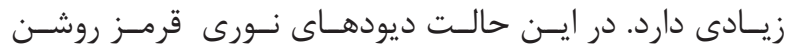

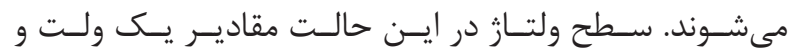

${ }^{7}$ Receiver

${ }^{8}$ Transmitter

${ }^{9}$ Infrared

${ }^{10}$ Serial communication

${ }^{11}$ Action

${ }^{12}$ Reward

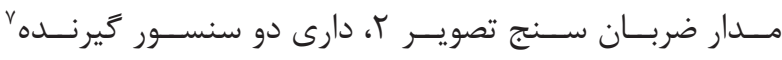

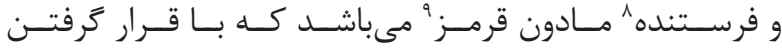

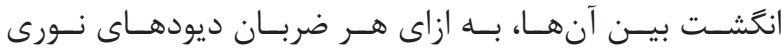

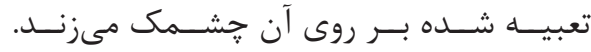

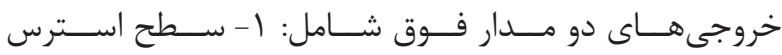

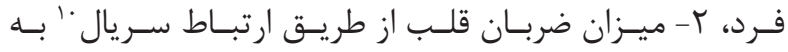

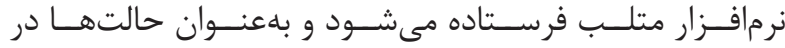

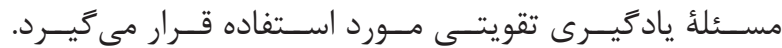

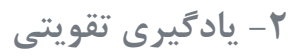

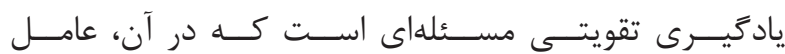

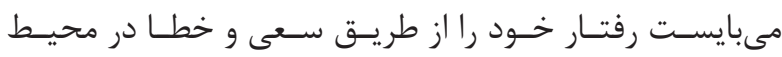

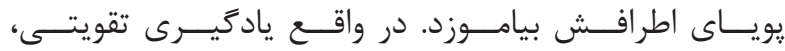

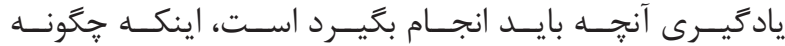

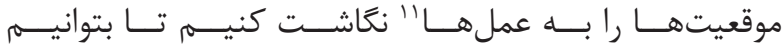

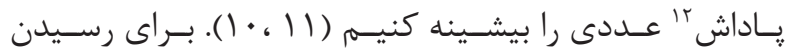

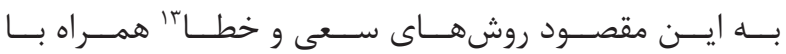

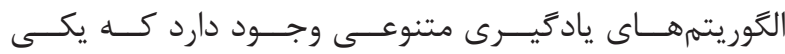

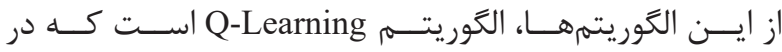

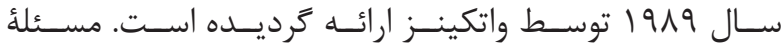

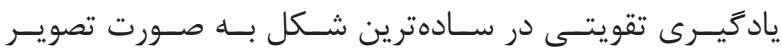

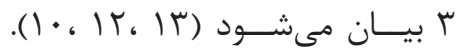

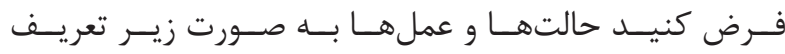
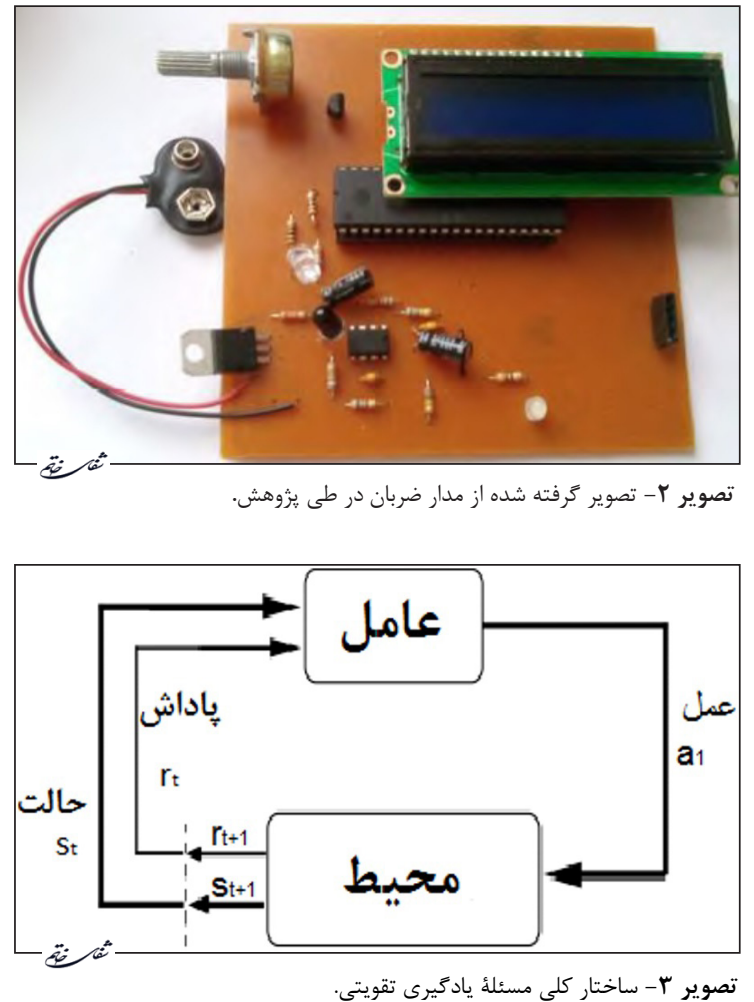
${ }^{13}$ Try and error
${ }^{14}$ State
${ }^{15}$ Voltage level
${ }^{16}$ Excitation
${ }^{17}$ Activity 
T-F

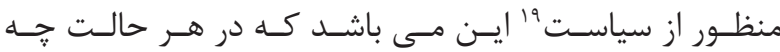

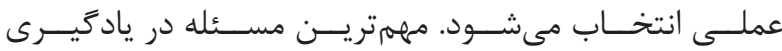

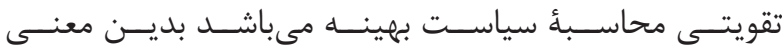

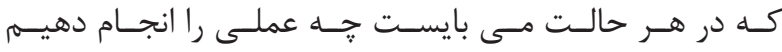

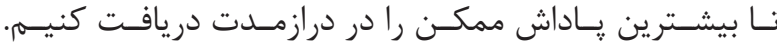

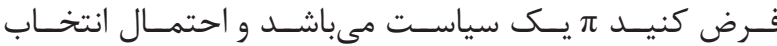

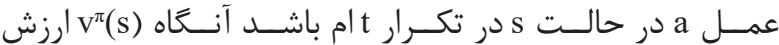

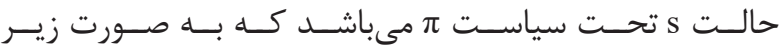

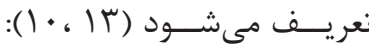

$$
V^{\pi}(s)=E_{\pi}\left\{R_{t} \mid s_{t}=s\right\}=
$$$$
E_{\pi}\left\{\sum_{k=0}^{\infty} \gamma^{k} r_{t+k+1} \mid s_{t}=s\right\}
$$

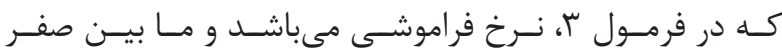

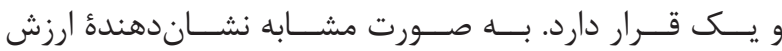

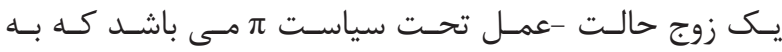

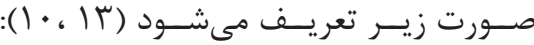

$$
\begin{array}{r}
Q^{\pi}(s, a)=E_{\pi}\left\{R_{t} \mid s_{t}=s, a_{t}=a\right\}= \\
E_{\pi}\left\{\sum_{k=0}^{\infty} \gamma^{k} r_{t+k+1} \mid s_{t}=s, a_{t}=a\right\}
\end{array}
$$

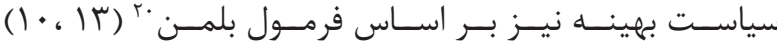

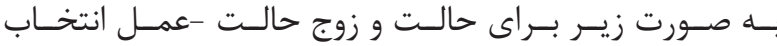

$V^{*}(s)=\max V^{\pi}(s)=$

$\max _{a \in A(s)} \sum_{s^{\prime}} P_{S S^{\prime}}^{\pi}\left[R_{S S^{\prime}}^{a}+\gamma V^{*}\left(s^{\prime}\right)\right]$

مى شــــود:

$$
\begin{aligned}
& Q^{*}(s, a)=\max _{\pi} Q^{\pi}(s, a)= \\
& \max _{a \in A(s)} \Sigma_{S^{\prime}} P_{S S^{\prime}}^{a}\left[R_{S S^{\prime}}^{a}+\right. \\
& \left.\gamma \max _{a^{\prime}} Q^{*}\left(s^{\prime}, a^{\prime}\right)\right]
\end{aligned}
$$

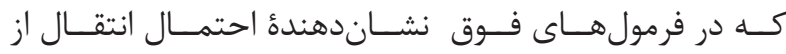

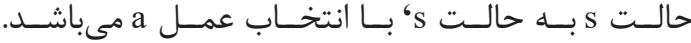

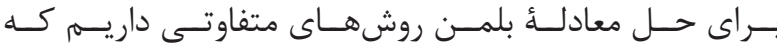

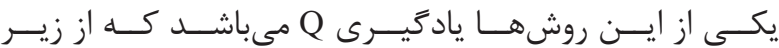

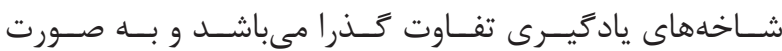

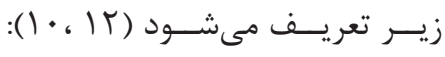

$$
\begin{array}{r}
Q\left(s_{t}, a_{t}\right)=Q\left(s_{t}, a_{t}\right)+ \\
\alpha\left[r_{t+1}+\gamma Q \max _{a}\left(s_{t+1}, a\right)-\right. \\
\left.Q\left(s_{t}, a_{t}\right)\right]
\end{array}
$$

\begin{tabular}{|c|c|c|c|}
\hline$v \leq 1$ & $1<v<2$ & $2 \leq v$ & سطح ولتاز \\
\hline حالت r & حالت r & حالت 1 & حالت \\
\hline
\end{tabular}

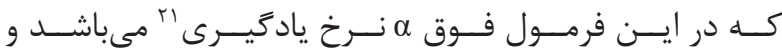

${ }^{18}$ Dynamic environment

${ }^{19}$ Policy

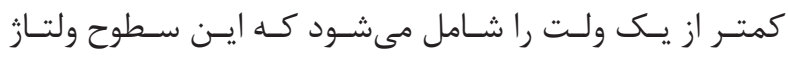

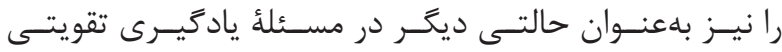

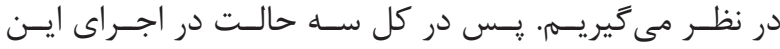

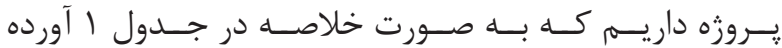

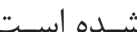

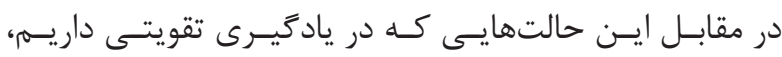

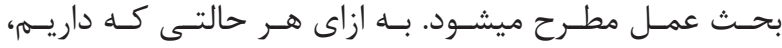

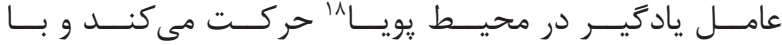

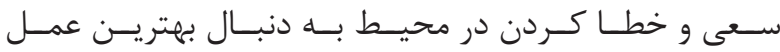

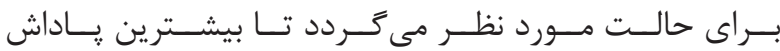

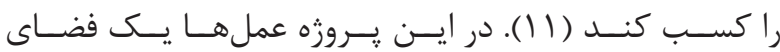

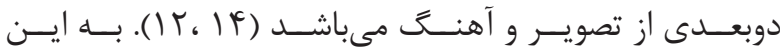

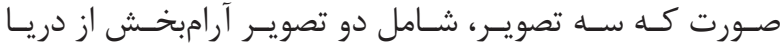

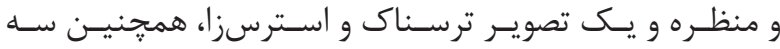

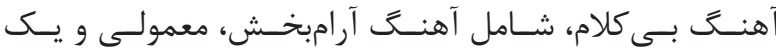

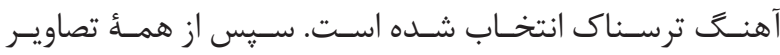

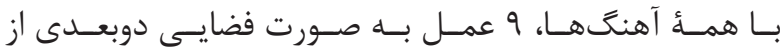

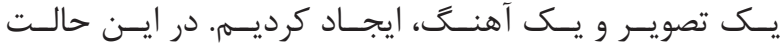

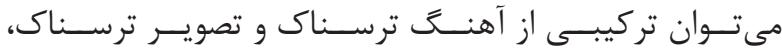

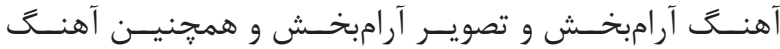

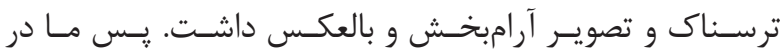

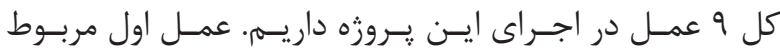

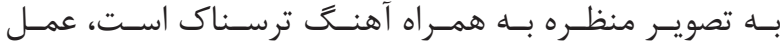

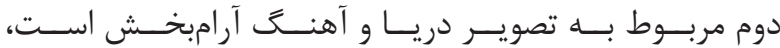

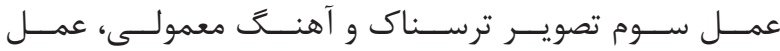

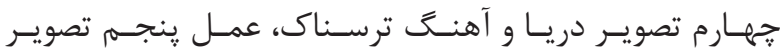

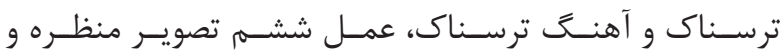

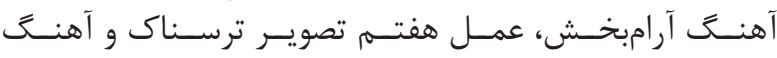

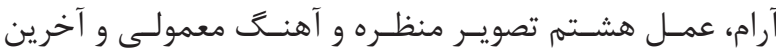

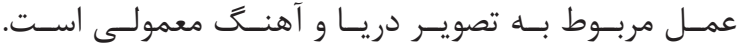

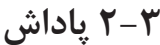

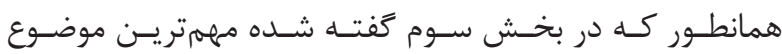

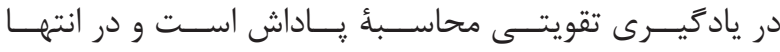

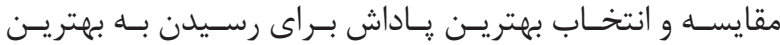

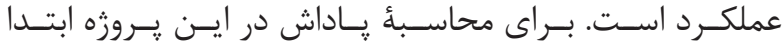

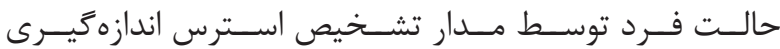

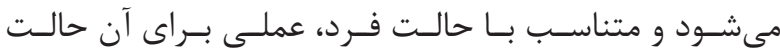

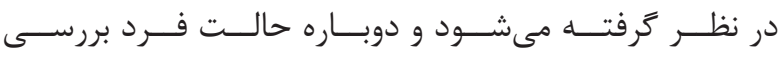

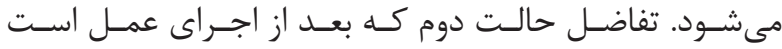

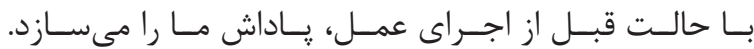

${ }^{20}$ Bellman

${ }^{21}$ Learning rate 


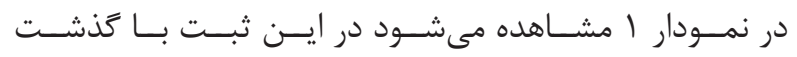

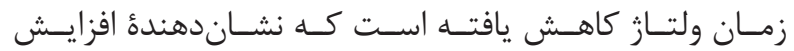

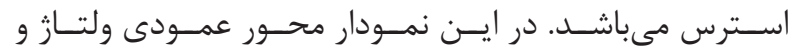

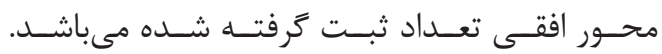

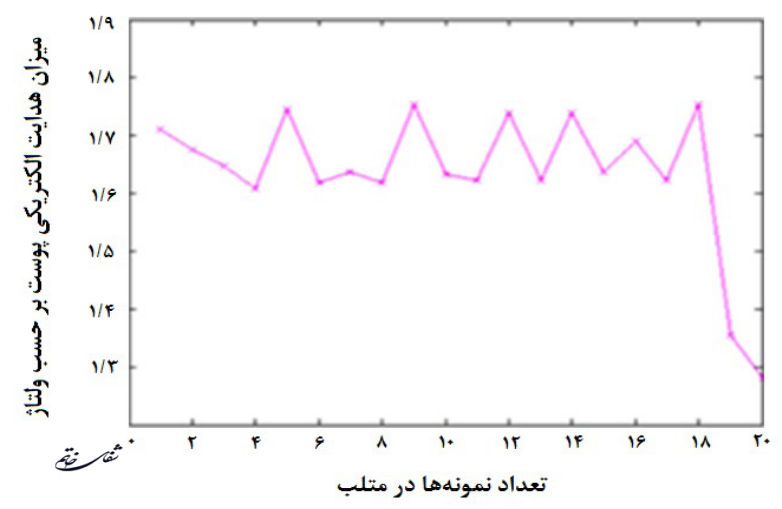

نمودار ا- نمونهاى از ثبت ميزان هدايت الكتريكى يوست بر حسب ولتاز به تعداد نمونهها

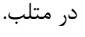

حـال يــس از دريافــت وضعيــت اســترس فــرد و تعييــن

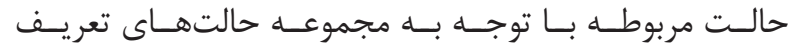

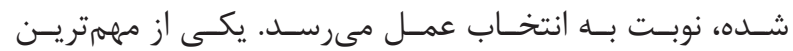

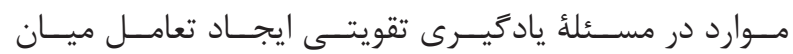

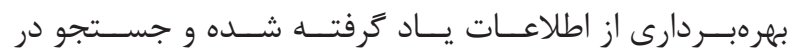

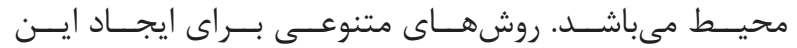

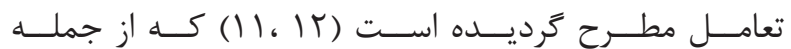

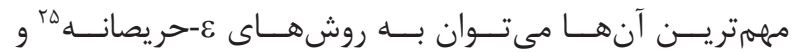

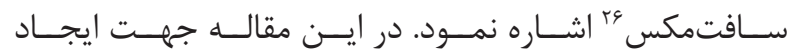

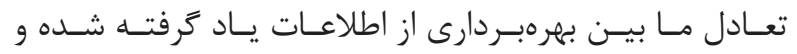

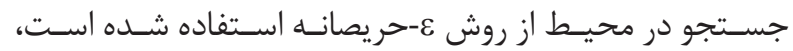

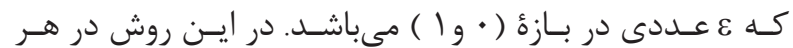

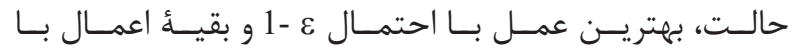

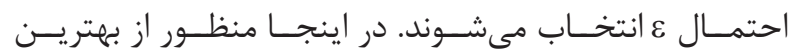

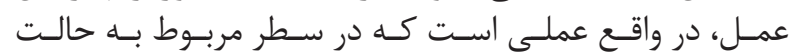

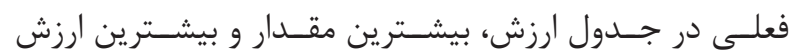

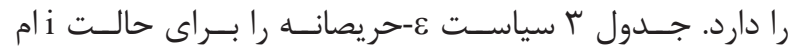

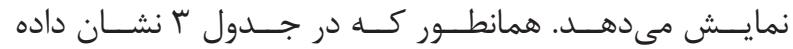

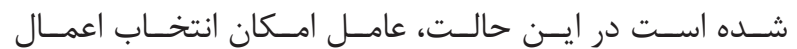

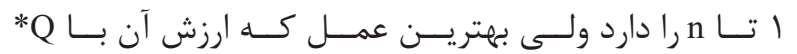

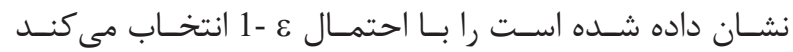

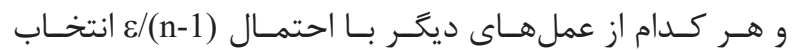

جدول r- تشريح سياست ع-حريصانه با توجه به ارزشها.

\begin{tabular}{|c|c|c|c|c|}
\hline عمل n & عمل j & عمل r & عمل & \\
\hline $\mathrm{Q}\left(\mathrm{s}_{\mathrm{i}}, \mathrm{a}_{\mathrm{n}}\right)$ & $Q^{*}\left(s_{i}, a_{j}\right)$ & $\mathrm{Q}\left(\mathrm{s}_{\mathrm{i}}, \mathrm{a}_{2}\right)$ & $Q\left(s_{i}, a_{1}\right)$ & حالت i ام \\
\hline$\varepsilon$ & \multirow{2}{*}{$1-\varepsilon$} & $\varepsilon$ & $\varepsilon$ & احتمال \\
\hline$\overline{n-1}$ & & $\overline{n-1}$ & $\overline{n-1}$ & انتخاب عمل \\
\hline
\end{tabular}

\section{${ }^{22}$ Elements}

${ }^{23}$ Serial port

${ }^{24}$ Cell
مقـــدارى در بـــازه ( • و ا ) دارد.

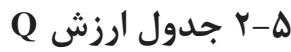

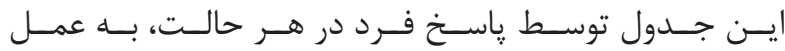

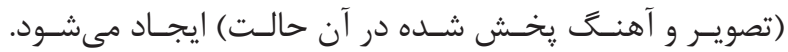

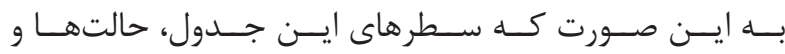

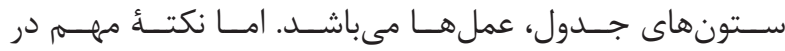

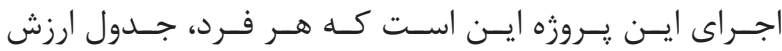

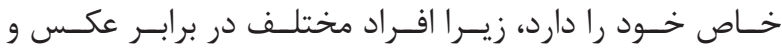

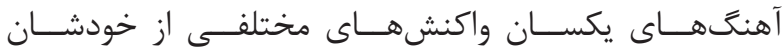

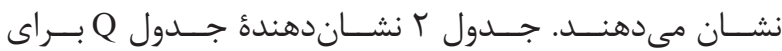

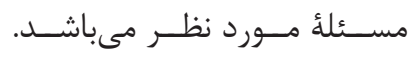

\begin{tabular}{|c|c|c|c|}
\hline عمل 9 9 & عمل r & عمل I & $\mathbf{Q}(\mathrm{s}, \mathrm{a})$ \\
\hline $\mathrm{Q}\left(\mathrm{s}_{1}, \mathrm{a}_{9}\right)$ & $\mathrm{Q}\left(\mathrm{s}_{1}, \mathrm{a}_{2}\right)$ & $\mathrm{Q}\left(\mathrm{s}_{1}, \mathrm{a}_{1}\right)$ & آرامش كامل \\
\hline $\mathrm{Q}\left(\mathrm{s}_{2}, \mathrm{a}_{9}\right)$ & $\mathrm{Q}\left(\mathrm{s}_{2}, \mathrm{a}_{2}\right)$ & $\mathrm{Q}\left(\mathrm{s}_{2}, \mathrm{a}_{1}\right)$ & طبيعى \\
\hline $\mathrm{Q}\left(\mathrm{s}_{3}, \mathrm{a}_{9}\right)$ & $\mathrm{Q}\left(\mathrm{s}_{3}, \mathrm{a}_{2}\right)$ & $\mathrm{Q}\left(\mathrm{s}_{3}, \mathrm{a}_{1}\right)$ & استرس \\
\hline
\end{tabular}

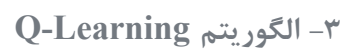

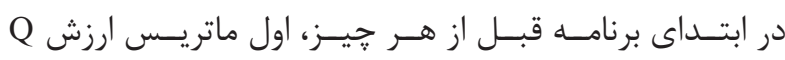

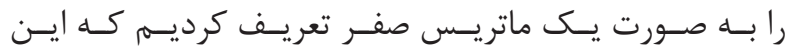

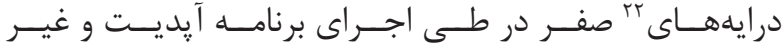

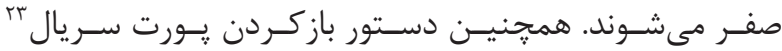

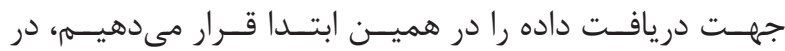

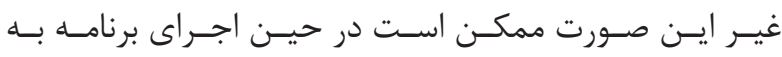

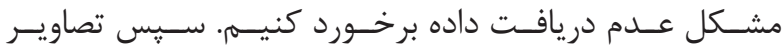

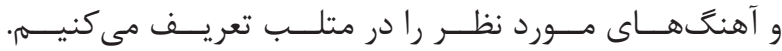

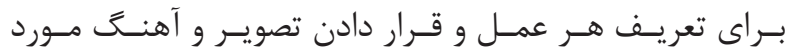

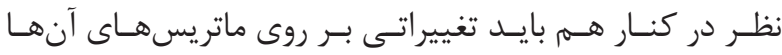

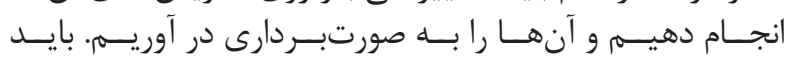

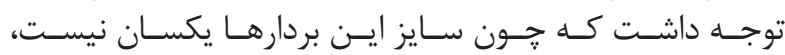

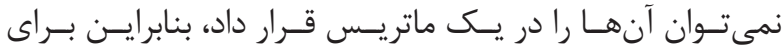

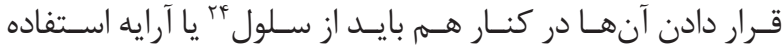

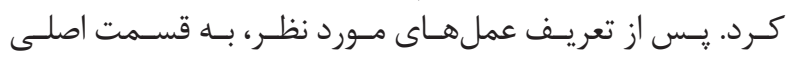

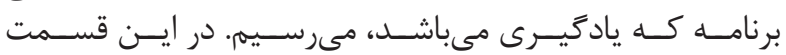

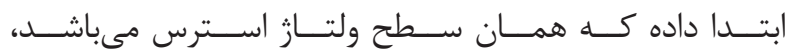

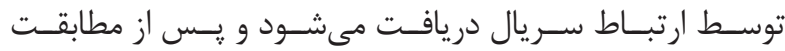

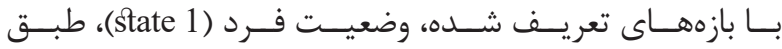

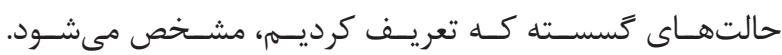

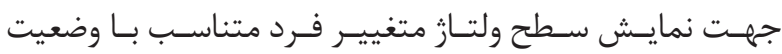

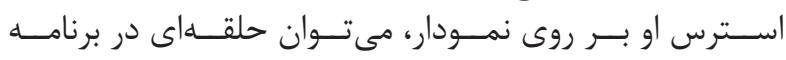

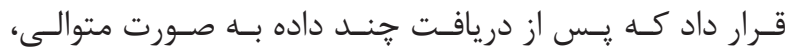

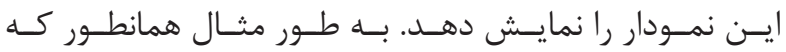

\footnotetext{
${ }^{25} \varepsilon$-greedy
}

${ }^{26}$ Softmax 


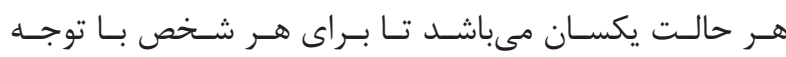

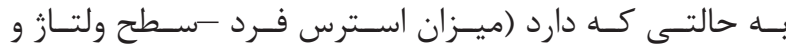

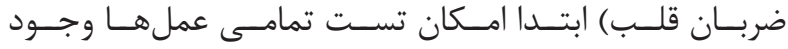

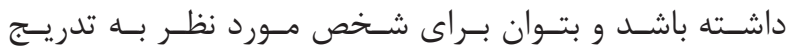

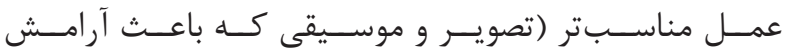

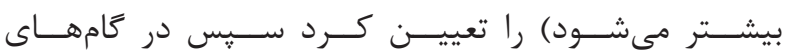

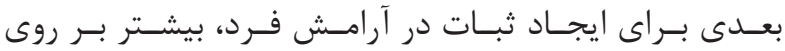

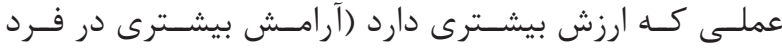

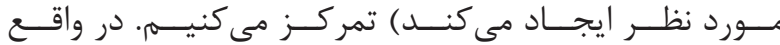

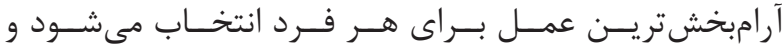

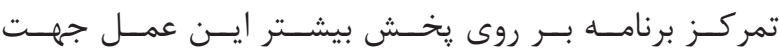

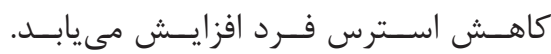

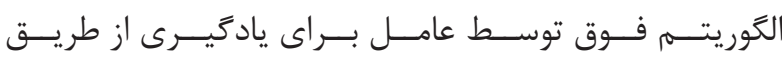

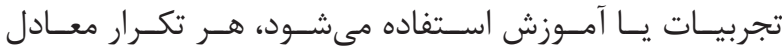

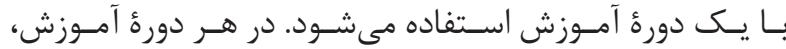

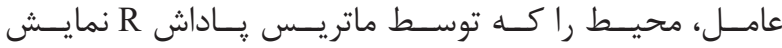

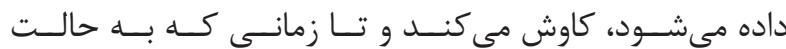

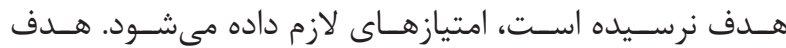

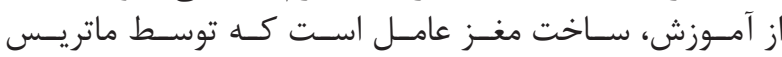

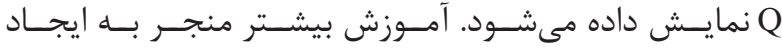

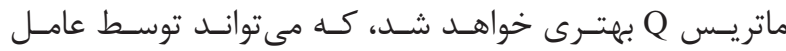

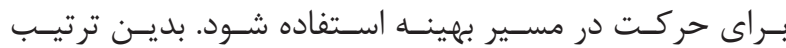

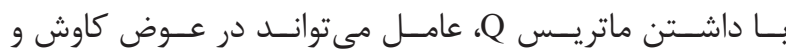

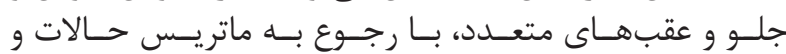

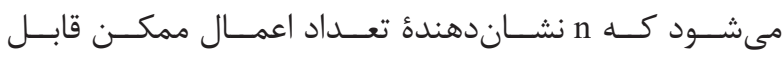

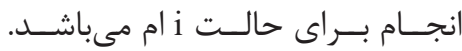

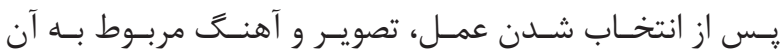

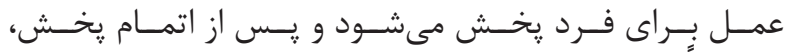

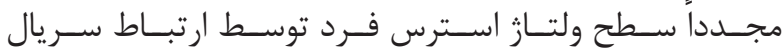

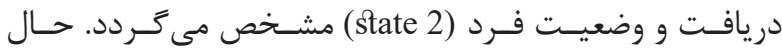

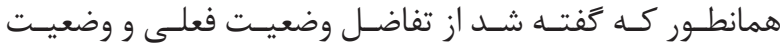

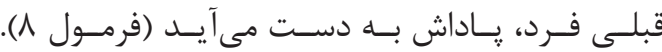

$$
\text { reward }=\mathrm{v}(2)-\mathrm{v}(1)
$$

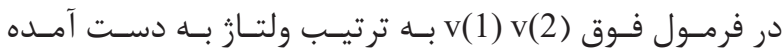

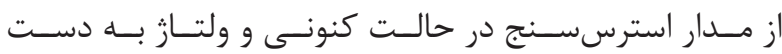

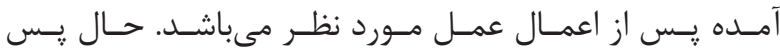

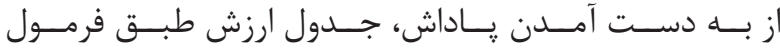

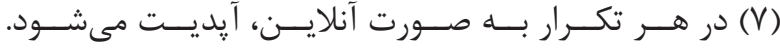

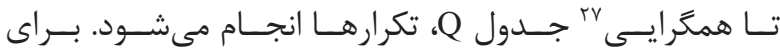

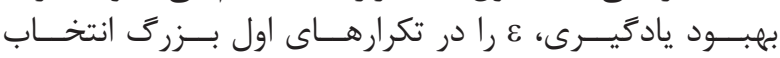

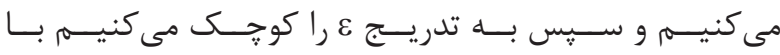

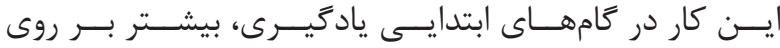

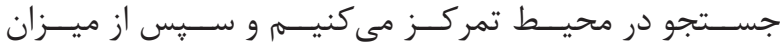

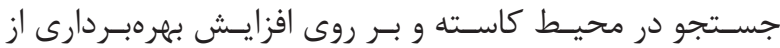

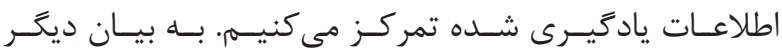

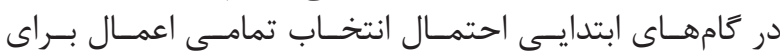

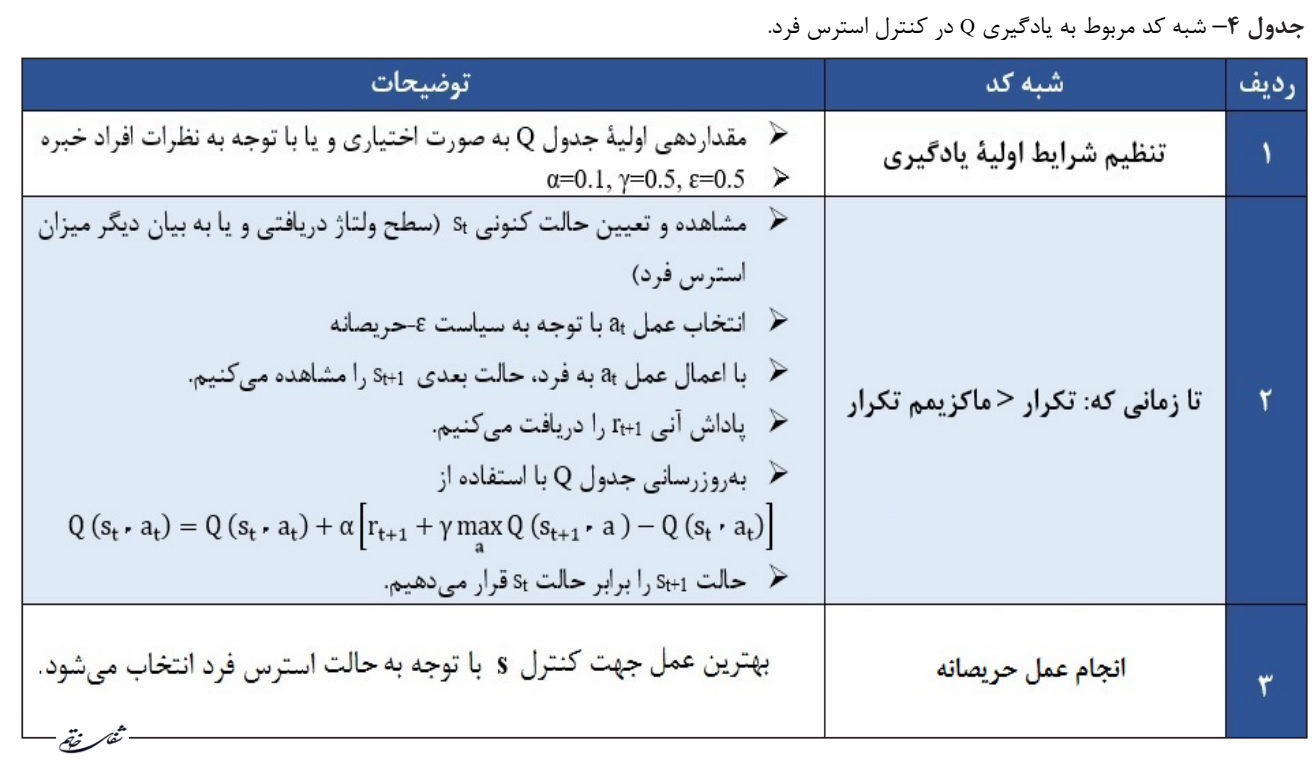

جدول ه- نمونه جدول Q به دست آمده از ارزش و عمل در اين تحقيق.

\begin{tabular}{|c|c|c|c|c|c|c|c|c|c|}
\hline عمل 9 & عمل 1 & عمل V & عمل 9 & عمل ه & عمل P & عمل ب & عمل Y & عمل 1 & \\
\hline . & سזו"ן. & $-\cdot \mid \cdot t \cdot \omega$ & $1 / \cdot \cdot r v$ & -.19919 & $-\cdot / \cdot 11 r$ & $-\|_{\mu} \mu \Delta$. & $\cdot \mid \lambda \cdot \mu q$ & $-.19 \mathrm{~V} \mid 1$ & آرامش كامل \\
\hline & $\cdot / 191 \Delta$ & $\cdot \cdot V V G$ & 1.1 .19$. & $-1 / \mu \mid \cdot 9$ & $\cdot \cdot$ VAr &.$- / 4 q \mu \wedge$ & $1 / r \cdot V \Lambda$ & $-\cdot \mid \cdot \Delta \mu \psi$ & طبيعى \\
\hline $.199 \mathrm{VY}$ & - DHYY & $\cdot \mid \Delta \Delta Q 1$ & 1/DGKT & $\cdot \mid \lambda \mu \nu$ & $\cdot \mid \cdot \cdot 1 \mathrm{~V}$ & $-\cdot \mid \cdot V \Delta \mu$ & - AVQ. & 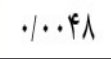 & استرس \\
\hline
\end{tabular}




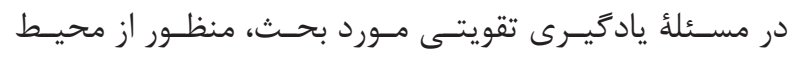

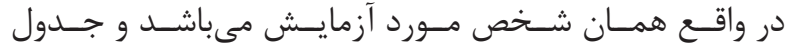

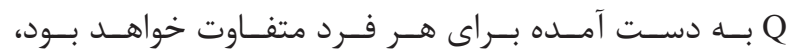

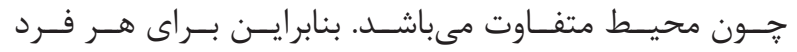

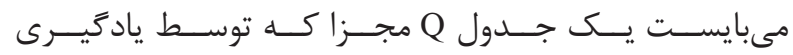

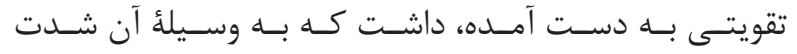

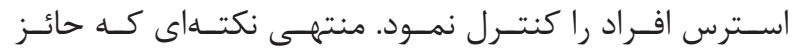

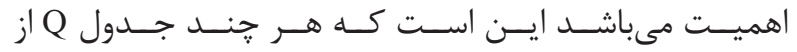

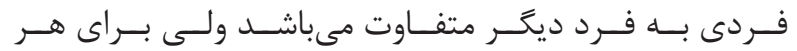

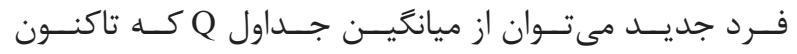

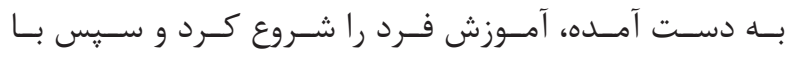

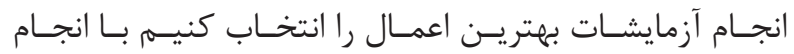

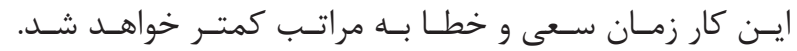

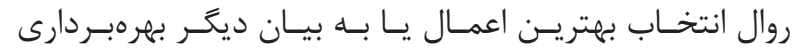

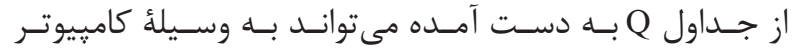

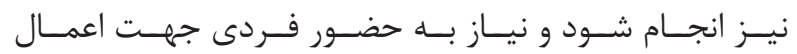

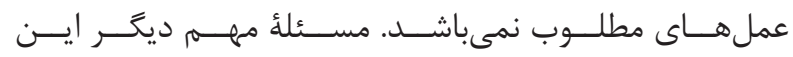

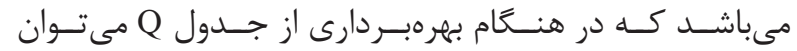

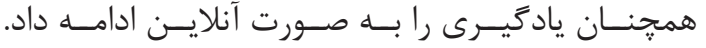

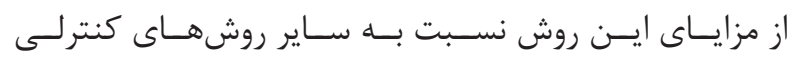

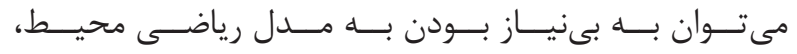

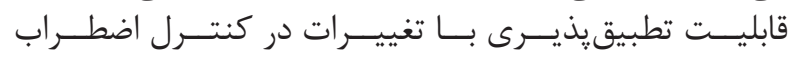

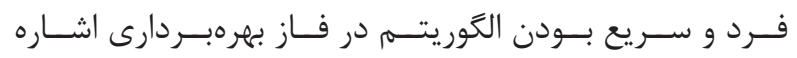

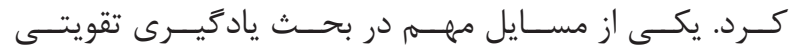

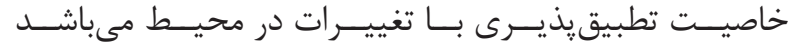

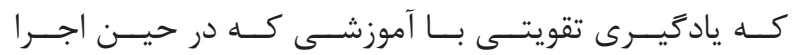

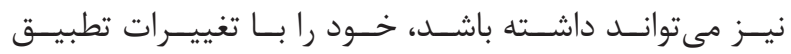

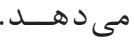

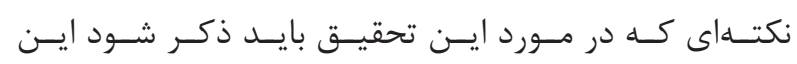

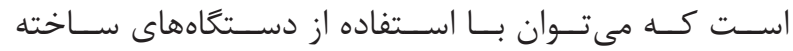

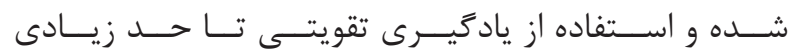

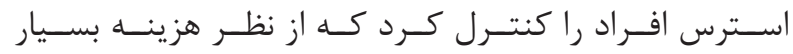

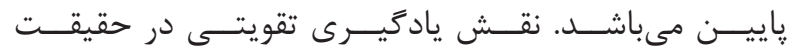

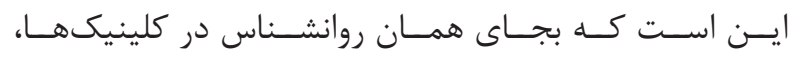

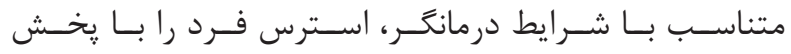

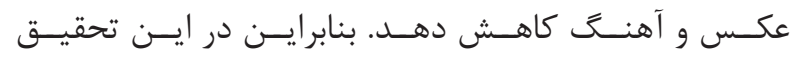

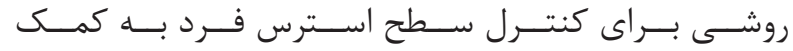

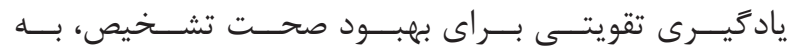

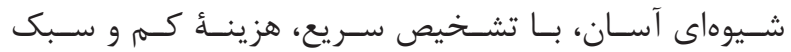

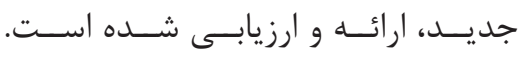

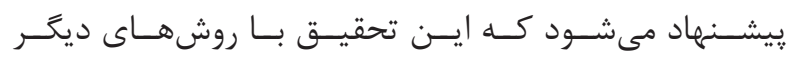

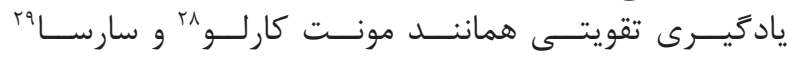

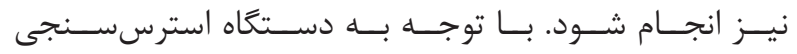

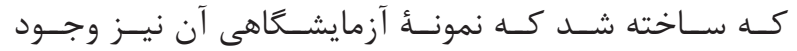

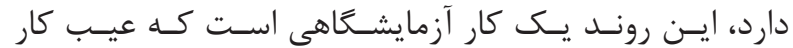

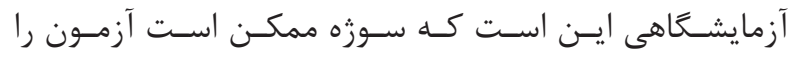

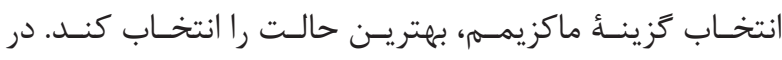

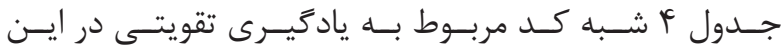

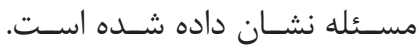

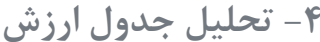

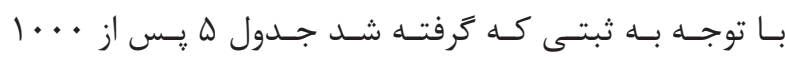

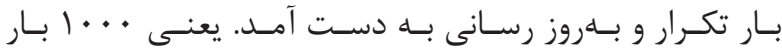

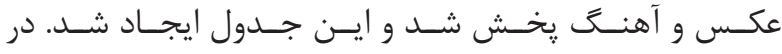

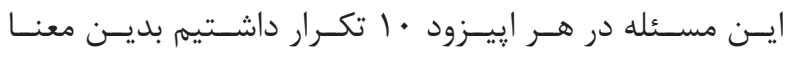

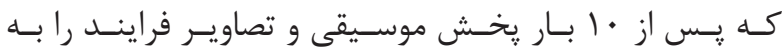

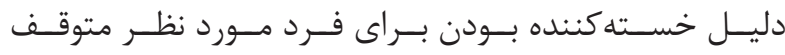

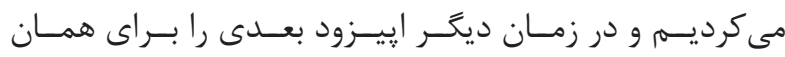

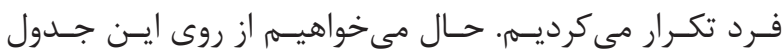

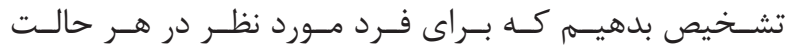

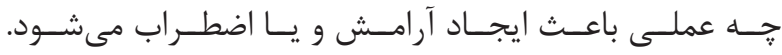

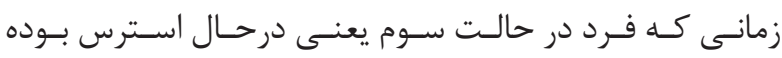

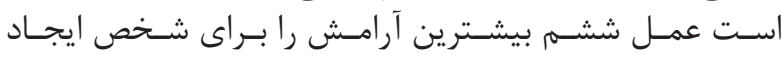

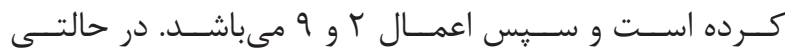

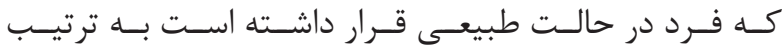

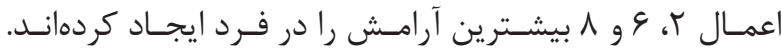

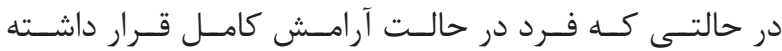

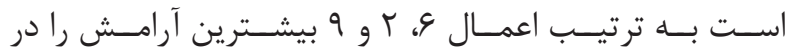

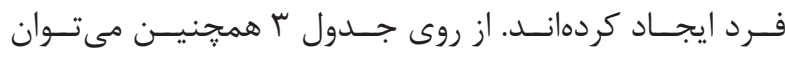

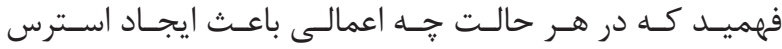

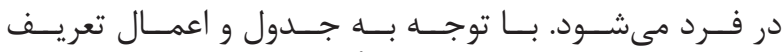

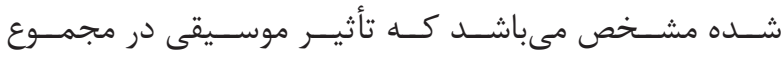

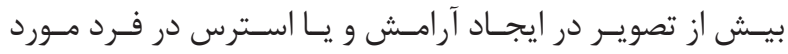

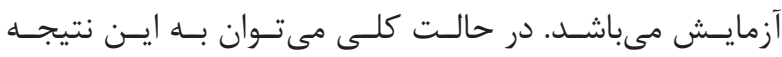

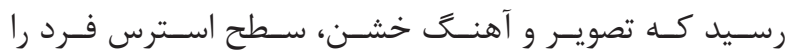

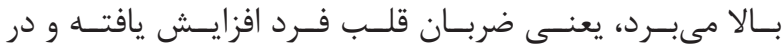

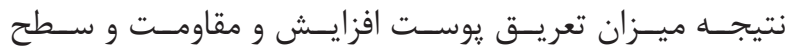

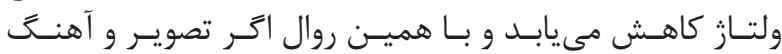

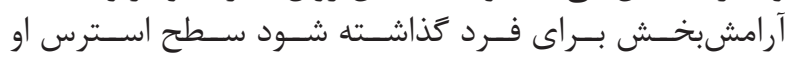

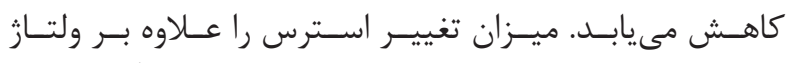

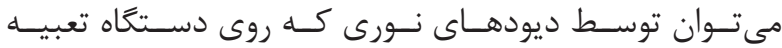
شــده، مشــاهده كـــرد.

\section{بحث و نتيجهَ كيرى}

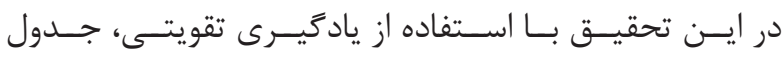

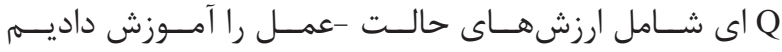

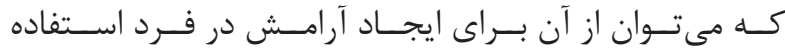

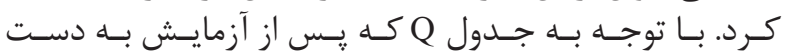

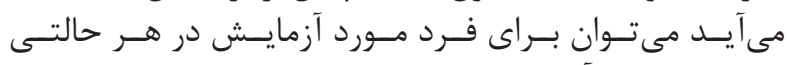

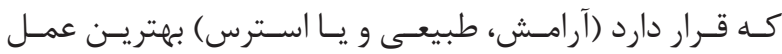

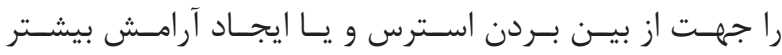

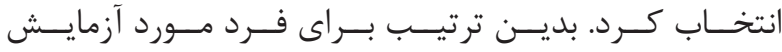

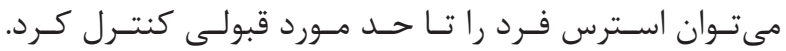




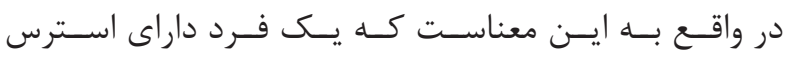

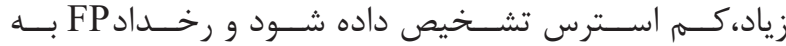

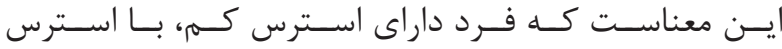

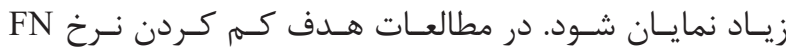

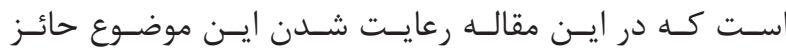
اهميـت اسـت كت د است

1. Fabiani M, Gratton G, Coles M, Federmeier KD. Event-related brain potentials: methods, theory and applications. cacioppo $\mathrm{j}$, tassinary $\mathrm{lg}$. handbook of psychophysiology. 3rd ed. Cambridge University Press. 2007; p.85-111.

2. Pedrotti M, Mirzaei MA, Tedesco A, Chardonnet JR, Mérienne F, Benedetto S, et al. Automatic stress classification with pupil diameter analysis. J HumanComputer Interaction. 2014; 30(3): 220-36.

3. Cook AE, Hacker DJ, Webb AK, Osher D, Kristjansson SD, Woltz DJ, et al. Lyin'eyes: ocular-motor measures of reading reveal deception. J Exp Psychol Appl. 2012; 18(3): 301-13.

4. Pavlidis I, Levine J. Thermal image analysis for polygraph testing. IEEE Eng Med Biol. 2002; 21(6): 56-64.

5. Lykken DT, Vemables PH. Direct measurement of skin conductance: a proposal for standardization. J Psychophysiology. 1971; 8(5): 656-72.

6. Fredrickson M, Ohman A. Cardiovascular and electrodermal responses conditioned to fear- relevant stimuli. J Psycophysiology. 1979; 16(1): 1-7.

7. Abdullah AA, Hassan UH. Design and development

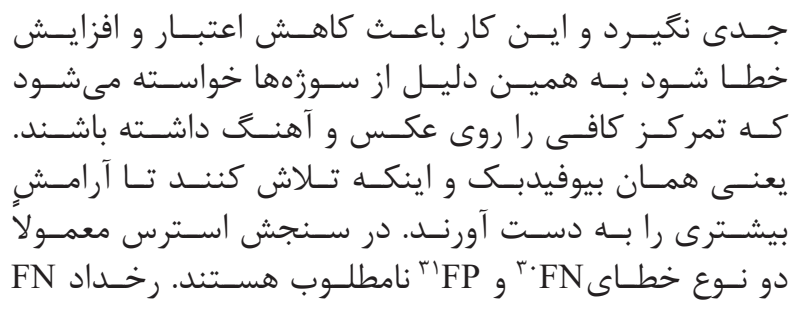

منابع

of an ESI kit. IEEE Conference on Student. 2012; 253-7.

8. Choi J, Gutierrez-Osuna R. Using heart rate monitors to detect mental stress. Body Sensor Networks. 2009; p. 219-23

9. van Dooren M, Janssen JH. Emotional sweating across the body: comparing 16 different skin conductance measurement locations. Physiology \& Behavior. 2012; 106(2): 298-304.

10. Richard S, Sutton RS, Barto AG. Reinforcement learning: an introduction. Cambridge: MIT Press. 1998.

11. Kaelbling LP, Littman ML, Moore AW. Reinforcement learning: a survey. JAIR. 1996; 4: 237-85.

12. Gosavi A. Reinforcement learning: a tutorial survey and recent advances. Informs J Computing. 2009; 21(2): 178-92.

13. Kakade SM. On the sample complexity of reinforcement learning. $\mathrm{PhD}$ thesis. University of London Gatsby Computational Neuroscience Unit. 2003.

14. Gomez P, Danuser B. Affective and physiological response to environmental noise and music. J Psychophysiology. 2004; 53(2): 91-103.

\footnotetext{
${ }^{30}$ False negative

${ }^{31}$ False positive
} 